\section{MedienPädagogik}

www.medienpaed.com
Zeitschrift für

Theorie und Praxis der Medienbildung

ISSN 1424-3636

Themenheft Nr. 14: Qualitative Forschung in der Medienpädagogik

\title{
Erfahrungen an der Schnittstelle von Medienarbeit und Praxisforschung
}

Peter Holzwarth und Björn Maurer

\section{Abstract}

Der folgende Beitrag reflektiert die Verbindung von praktischer Medienarbeit und Forschung am Beispiel eines internationalen EU-Forschungsprojekts zum Thema Medien und Migration. Neben didaktischen Prinzipien und Konzepten für die aktive Medienarbeit im Forschungskontext geht es um die Kooperation von medienpädagogischer Begleitung (MB) und wissenschaftlicher Begleitung (WB) sowie um deren spezifische Kompetenzen.

\section{Das internationale EU-Forschungsprojekt CHICAM}

CHICAM - "Children in Communication about Migration» war ein internationales EU-Praxisforschungsprojekt, das von 2001 bis 2004 an der Pädagogischen Hochschule Ludwigsburg und anderen Universitäten und Institutionen in London, Rom, Athen, Stockholm und Utrecht durchgeführt wurde. Junge Migrantinnen und Migranten aus verschiedenen Ländern der Erde, die in Deutschland, Italien, Griechenland, Schweden, Gross-Britannien und in den Niederlanden leben, produzierten im Kontext von lokalen, wöchentlich stattfindenden «CHICAM-Clubs» Fotos und Videos und gaben so Einblicke in ihre Lebenswelten (Themenbereiche: Familie, Peergroup, Schule, Mediennutzung). Die Produktionen, die von erfahrenen Medienpädagogen begleitet wurden, konnten jeweils von den Kindern und Jugendlichen der anderen Länder über eine Intranet-Plattform angeschaut und kommentiert werden. Zugleich wurden die Produktionen der Kinder unterschiedlichen Öffentlichkeiten zugänglich gemacht, um im sozialen Umfeld, in lokalen und in politischen Institutionen ein stärkeres Bewusstsein
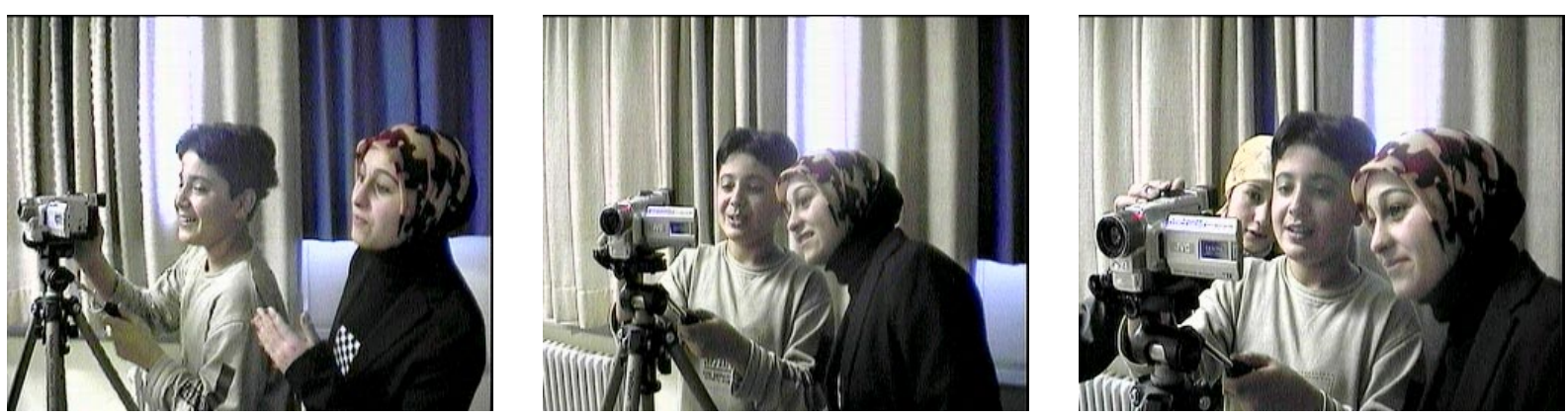

Abbildung 1: Junge Migranten und Migrantinnen im «CHICAM-Club» 
für die Lage von Kindern aus Migrations- und Fluchtkontexten zu schaffen. Das Projekt verfolgte das Ziel, die Potenziale aktiver Medienarbeit für interkulturelle Kommunikation, Integration und Reflexion zu untersuchen (Holzwarth \& Maurer 2003; de Block et al. 2004).'

CHICAM knüpfte an Erfahrungen des internationalen Projekts VideoCulture (Niesyto 2003) an und verband eine ethnografisch-lebensweltliche Dimension mit einer medienpädagogischen Dimension. Die ethnografische Dimension diente

\begin{tabular}{|l|l|}
\hline \multicolumn{2}{|c|}{ Projektdimensionen von CHICAM } \\
\hline Forschungsdimension & $\begin{array}{l}\text { - Lebenswelten von jungen Migranten und Migrantinnen erforschen (Peer- } \\
\text { group, Familie, Schule, Mediennutzung) } \\
\text { - Entwicklung nicht-sprachlicher Zugänge und Ausdrucksmöglichkeiten ergän- } \\
\text { zend zum verbalen Ausdruck, Weiterentwicklung visueller Methoden } \\
\text { - Möglichkeiten und Grenzen interkultureller Kommunikation mit dem Intranet } \\
\text { erforschen } \\
\text { - Weiterentwicklung medienpädagogischer Konzepte für interkulturelle Kon- } \\
\text { texte }\end{array}$ \\
\hline Praktische Dimension & $\begin{array}{l}\text { - Medienpädagogische Begleitung von Eigenproduktionen (Video und Foto- } \\
\text { grafie) im Rahmen von Medienclubs } \\
\text { - Vermittlung von medialen, sozialen und reflektiven Kompetenzen }\end{array}$ \\
\hline Politische Dimension & $\begin{array}{l}\text { - Über das Öffentlichmachen von Eigenproduktionen Sensibilität und Bewusst- } \\
\text { sein für Kinder/Jugendliche aus Migrations- und Fluchtkontexten entwickeln } \\
\text { Die Stimmen und Themen von Kindern aus Flucht- und Migrationskontexten } \\
\text { in der (politischen) Öffentlichkeit hörbar und sichtbar machen ("give them a } \\
\text { voice») }\end{array}$ \\
\hline
\end{tabular}

Tabelle 1: Projektdimensionen von CHICAM im Überblick

der Erkundung der Lebenswelten der Kinder und Jugendlichen mittels Medienproduktionen, teilnehmender Beobachtung, Einzel- und Gruppengesprächen. Im Rahmen der medienpädagogischen Dimension wurden vor allem Chancen einer audiovisuellen Kommunikation mittels Fotografie, Video und Internet erprobt und erforscht. Ausserdem wurden medienpädagogische Praxiskonzepte für Kinder und Jugendliche mit Migrationskontext weiterentwickelt (vgl. Maurer 2004). Die visuellen Ausdrucksformen Fotografie und Video stellten eine wichtige Ergänzung zu verbalen Erhebungsmethoden dar. Dies war insofern hilfreich, als die Kinder und Jugendlichen sich noch im Prozess des Zweitspracherwerbs befanden. Grundlage von Interviews zu den Bereichen Peergroup, Familie, Schule und Mediennutzung waren Fotos, die die Kinder zu den genannten Themen mit Einwegkameras selbst produziert hatten (photo-elicitation). 
Im Rahmen des Projekts kamen Forscher (Pädagogik, Ethnologie, Psychologie, Medienwissenschaft) und Praktiker (schulische Medienarbeit, ausserschulische Medienarbeit, Videokunst) aus verschiedenen Kontexten zusammen. In jedem Land entstand für die Dauer eines Jahres ein Medienclub (CHICAM Club), der bis auf eine Ausnahme - an Schulen angesiedelt war. ${ }^{2}$ Geleitet wurden die Clubs von einer medienpädagogischen Begleitung (MB) und einer wissenschaftlichen Begleitung (WB). Die MB war schwerpunktmässig für die Medienarbeit im Club zuständig, die WB für die Forschung.

Einige Partner verfügen über spezielle Erfahrungen im Bereich Migrationsarbeit und -Forschung, andere dagegen haben ihre Arbeitsschwerpunkte eher im Bereich Medienpädagogik und Medienforschung. Dadurch ergab sich eine interessante Kooperationssituation, in der die unterschiedlichen Partner von der jeweiligen Expertise der anderen profitieren konnten.

In allen Ländern wurden visuelle und verbale Daten zu den genannten Themenfeldern Peergroup, Familie, Schule und Medien/visuelle Kommunikation erhoben, wobei jeweils zwei Projektpartner schwerpunktmässig für einen Bereich zuständig waren. Die Kommunikation zwischen den Projektpartnern fand auf internationalen Forschungstreffen und bilateralen Treffen statt. Zusätzlich wurde eine CHICAM Researcher-Intranetplattform für den gegenseitigen Austausch genutzt.
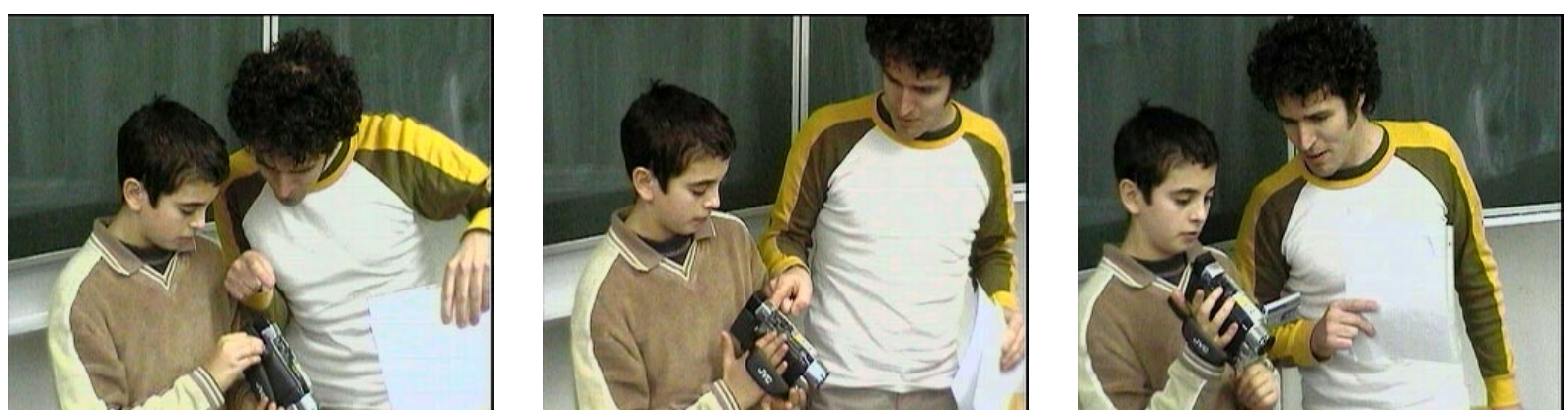

Abbildung 2: Aktive Medienarbeit in den «CHICAM-Clubs»

\section{Didaktische Prinzipien aktiver Medienarbeit mit Kindern aus Migrationskontexten im Rahmen des Projekts CHICAM}

Die im Folgenden dargestellten didaktischen Prinzipien gelten für jede Art von aktiver Medienarbeit mit Zielgruppen, deren sprachliche Kompetenzen noch im Prozess der Entwicklung begriffen sind. Unabdingbar sind sie im Kontext von Forschung, wenn es nicht nur um die Erstellung von Produkten und die Initiierung von Lernprozessen geht, sondern auch darum, über die Produkte subjektnahe Zugänge zur Lebenswelt der Kinder und Jugendlichen zu bekommen. 
Aktive Medienarbeit bzw. aktive Videoarbeit war die zentrale Arbeitsform in den CHICAM Clubs. Aufgrund der spezifischen Zielgruppe musste vom klassischen Muster, d. h. von einer Orientierung am professionellen Produktionsverlauf «Exposee, Storyboard, Drehplan, Aufnahmephase, Schnitt», abgewichen werden. Dies hätte von den jungen Produzenten/-innen gleich zu Beginn ein zu hohes Mass an Planung, Verbalisierung und Reflexion verlangt, was wiederum die Bereitschaft zur Teilnahme verringert hätte. Stattdessen haben wir bereits bei der Planung der Projektpraxis didaktische Prinzipien definiert, die ein niederschwelliges Arbeiten mit schnellen Erfolgserlebnissen ermöglichen sollten. Wir entwickelten Konzepte, die zunächst auf verbal-reflexive Arbeitsphasen verzichten und den Produzenten/-innen sehr schnell die Möglichkeit geben, spielerische Erfahrungen mit dem Medium zu machen. Lernumgebungen mit klar definierten ästhetischen Problemstellungen sollten zum eigenständigen Entdecken einladen und medienästhetische Lernprozesse auf verschiedenen Ebenen anregen. Im Folgenden werden die didaktischen Prinzipien kurz umrissen.

\section{Lebensweltorientierung}

Nicht nur die Themen und Interessen der Subjekte müssen in diesem Zusammenhang berücksichtigt werden, sondern auch ihr sozio-ökologisches Umfeld, ihre Alltags- und Lieblingsorte. Wir haben gute Erfahrungen mit Drehorten gemacht, die eine besondere Bedeutung für die Kinder hatten. Ideen für Filmgeschichten entstanden spontan vor Ort. Statt umfangreichen Vorarbeiten mit Drehbuch etc. war lediglich der Impuls «Was könnte hier passieren?» nötig, um kurze narrative Handlungseinheiten zu entwerfen und filmisch umzusetzen.

Ein weiterer wichtiger Bestandteil der Lebenswelt von Kindern mit Migrationshintergrund sind ihre Medienerfahrungen. Baacke u. a. (1990) prägten in diesem Zusammenhang den Begriff der «Medienwelten». Eine lebensweltorientierte Medienarbeit mit Kindern und Jugendlichen knüpft auch an den individuellen Medienbiographien der Subjekte an. Es kann sinnvoll sein, die Medienbiographien zum Gegenstand von Medienproduktionen zu machen, um als Pädagoge und Forscher Einblicke in Mediennutzungsgewohnheiten und -präferenzen zu bekommen (vgl. Schell 1999 S. 114).

\section{Problemorientierung}

Lernen ist nach unserem Verständnis ein aktiver, vom Individuum ausgehender kognitiver Prozess, in welchem Wissensbestände, Handlungs- und Problemlösekompetenzen auf der Basis von Vorwissen, Erwartungen und subjektiven Erfahrungen sukzessive aufgebaut werden (vgl. z. B. Friedrich u. a. 1997 S. 8). Wir gehen ferner davon aus, dass Kinder und Jugendliche durch ihre Medienerfahrungen bereits über ein hohes Mass an medienästhetischem und mediendramaturgischem Wissen verfügen (vgl. Buckingham u. a. 1995 S. 221). Dieses 
Wissen ist zunächst noch in passiver Form vorhanden und kann im Rahmen medienpädagogisch begleiteter Lernprozesse in aktives und verfügbares Wissen transformiert werden. Der didaktisch-methodische Weg, auf welchem sich diese Transformation vollziehen kann, ist selbsttätiges, problemorientiertes Lernen. Um eine Problemstellung lösen zu können, sind verschiedene Teilkompetenzen und Wissenseinheiten erforderlich, die im Rahmen des Lösungsprozesses erworben werden (vgl. Friedrich u. a. 1997 S. 95). Didaktisch gesehen werden Teilkompetenzen und Wissen nicht durch ein Curriculum von Lernzielen organisiert, sondern sie sind den einzelnen Problemstellungen inhärent.

\section{Anschaulichkeit}

Bei der Gestaltung von Lernmaterialien hat es sich als sinnvoll erwiesen, in Anlehnung an den Deutsch-als-Fremdsprache-Unterricht (DaF) auf präsentative, interkulturell verstehbare Symbolsysteme zurückzugreifen. Durch Anschaulichkeit, die den Kindern einen Lerngegenstand unmittelbar zugänglich macht, konnten komplizierte Erklärungen und Belehrungen reduziert werden. Bei der Verwendung von Wort-Bild-Karten bspw. hatten die Kinder die Wahl zwischen der diskursiven und der präsentativen Aneignungsform. Filmästhetische Strategien wurden in problemorientierten Lernumgebungen von den Kindern selbstständig angeeignet und anschliessend am gefilmten Material der Kinder gemeinsam auf den Begriff gebracht.

\section{Ästhetische Modelle nutzen}

Wichtig im didaktischen Gesamtarrangement war auch der Einsatz von ästhetischen Modellen. Ästhetische Modelle sind Beispielproduktionen, die bestimmte Sachverhalte und Lerninhalte veranschaulichen (z. B. die verschiedenen Macharten von Videoclips). Durch den Einsatz ästhetischer Modelle konnten verballastige Erklärungsphasen der medienpädagogischen Begleitung minimiert werden. Stattdessen sprachen die Modelle ihre eigene, anschauliche Sprache. Ästhetische Modelle können sowohl professionelle Produktionen, als auch audiovisuelle Materialien der Kinder selbst sein. Die didaktische Auswahl der Modelle sollte nach folgenden, im CHICAM Projekt bewährten Kriterien getroffen werden:

- Sie sollten die didaktisch gewünschten medienspezifischen Sachverhalte exemplarisch veranschaulichen können.

- Sie sollten zumindest teilweise aus dem (Medien-) Erfahrungs- und Präferenzbereich der Subjekte stammen (vgl. Lebensweltbezug).

- Die inhaltliche und ästhetische Qualität der ästhetischen Modelle sollte die voraussichtlich von den Kindern zu erzielende Qualität nicht zu weit übersteigen, um eventuelle Enttäuschungen zu vermeiden. 
- Stehen keine geeigneten ästhetischen Modelle zur Verfügung, kann die medienpädagogische Begleitung selbst ästhetische Modelle herstellen.

\section{Scaffolding/Fading}

Ausschliesslich selbstgesteuertes und kollektives Lernen - wie es die radikalen Konstruktivisten fordern - stösst insbesondere dann an Grenzen, wenn die Lernenden mit dieser Form des Lernens nicht vertraut sind (vgl. Dubs 1995).

Kinder und Jugendliche, die im Schulalltag vorwiegend mit Frontalunterricht konfrontiert werden, haben selten Gelegenheit, sich Kompetenzen anzueignen, die für die selbstständige Planung, Durchführung und Evaluation des Lernprozesses erforderlich sind. Aus diesem Grund kann selbstständiges Lernen zwar ein Ziel des Lernprozesses, nicht aber die zentrale Methode sein. Einen situationsadäquaten Wechsel zwischen Scaffolding, d. h. medienpädagogischen Impulsen und Hilfestellungen, und Fading, d. h. einer prozessgerechten Rücknahme der Unterstützung halten wir dagegen für ein entscheidendes didaktisches Prinzip für die Medienarbeit mit Kindern aus Migrationskontexten.

\section{Frustrationserfahrungen minimieren -} schnelle ästhetische Erlebnisse ermöglichen

Um die Kinder über einen längeren Zeitraum für Medienarbeit zu motivieren, war es u.a. wichtig, in möglichst kurzer Zeit Erfolgserlebnisse zu ermöglichen, d.h. vorzeigbare Ergebnisse zu erhalten. Der dramaturgische Bogen zwischen Materialerstellung und Fertigstellung durfte daher nicht überspannt werden. ${ }^{3}$ Es wurden insbesondere zu Beginn der Praxisphase überschaubare Einheiten geplant, die sich im zeitlichen Rahmen von 90 Minuten realisieren liessen. Bei komplexeren Projekten wie z. B. der digitalen Postproduktion eines Videofilms hat es sich als sinnvoll erwiesen, dass die medienpädagogische Begleitung das Video-Rohmaterial vorbereitet (z. B. Digitalisierung des Videomaterials, Vorauswahl der Sequenzen, Kürzen der Sequenzen). Die Kinder konnten somit aus einem begrenzten Materialpool auswählen, ohne sich in Detailentscheidungen zu verlieren. Auf diese Weise wurden Frustrationserfahrungen minimiert und schnelle ästhetische Feedbacks durch fertige Produkte ermöglicht.

\section{Didaktische Flexibilität und Offenheit}

Subjektorientierung verlangt die Bereitschaft der medienpädagogischen Begleitung, ggf. von eigenen Planungen abzuweichen und das pädagogische Konzept flexibel der Situation anzupassen. Sie betreibt gewissermassen medienpädagogische Aktionsforschung, indem sie kontinuierlich den Kenntnisstand, die Interessenlagen und die Motivation der Subjekte beobachtet, didaktische Arrangements entsprechend abstimmt und jeweils im Anschluss reflektiert, wie die Subjekte mit den medienpädagogischen Inputs umgegangen sind. Die me- 
dienpädagogische Begleitung im Kontext qualitativer Forschung muss sich als Lernender wahrnehmen, der seinen Lernerfolg unmittelbar an den pädagogischen Prozess rückkoppelt. Denn nur dann kommt es nicht zum Widerspruch zwischen der (notwendigen) didaktischen Struktur und den Prinzipien der Handlungsforschung.

\section{Einbezug von präsentativen Ausdrucksformen wie Theater und Tanz}

Subjektorientierte Didaktik greift körpersprachliche Kompetenzen auf, die bei Kindern und Jugendlichen aus Migrationskontexten häufig stark ausgeprägt sind. Wir halten eine Verknüpfung von medienpädagogischen Elementen mit Darstellungsformen und Übungen aus der Theaterpädagogik für sinnvoll. Mit Theaterpädagogik meinen wir an dieser Stelle nicht das klassische Einstudieren (Auswendiglernen) und Aufführen einer literarischen Vorlage, sondern das behutsame und durch entsprechende Übungen pädagogisch begleitete Sensibilisieren der Wahrnehmung des eigenen Körpers. Vorstellbar sind pantomimische Übungen, Spiele mit Bewegung und Tanz, Körper-und-Raum-Übungen sowie die gezielte Schulung von Gestik und Mimik.

\section{Forschung im Kontext praktischer Medienarbeit}

Forschung im Kontext praktischer Medienarbeit stellt für alle Beteiligten eine Herausforderung dar. Im Rahmen des Projekts wurden sowohl an die MB als auch an die WB hohe Anforderungen gestellt. In allen Partnerländern war die Suche nach einem Medienpädagogen mit adäquatem Kompetenzprofil sehr schwierig. In zwei Ländern musste nach kurzer Zeit eine qualifiziertere Person gefunden werden. Im Folgenden werden verallgemeinernd Anforderungsprofile für Forscher und Medienpädagogen im Kontext interkultureller medienpädagogischer Praxisforschung formuliert.

\section{Kompetenzen: Medienpädagogische Begleitung}

- Technische Kompetenzen (Filmschnitt, Kamerabedienung, Kameraeffekte, Videoformate, Filmstreaming über das Netz etc.)

- Die Fähigkeit Kinder/Jugendliche mit Migrationshintergrund bei der Produktion von Fotos und Videos medienpädagogisch zu unterstützen (im Spannungsfeld von Strukturierung (Projektziele) und Offenheit (Befähigung zum eigenständigen Arbeiten)

- Sensibilität für pädagogische Prozesse in der Gruppe (Störungen, Konflikte, Aussenseiter)

- Leiten von Gruppen (breites Methodenrepertoire, Gruppenspiele, mediale Übungen) 
- Antizipation von Verstehensmöglichkeiten im medienpädagogischen Kontext (Was können die Teilnehmenden verstehen? Wie muss ich vermitteln, damit sie verstehen können?)

- Visualisierungskompetenzen

- Motivierungskompetenzen

- Frustrationstoleranz

- Distanzierung vom eigenen Geschmack, Verzicht auf den Anspruch, sich mit dem Produkt ästhetisch und inhaltlich auch selbst als Medienpädagoge identifizieren zu können

\section{Gemeinsame Kompetenzen:}

- Erfahrung mit Kindern/Jugendlichen aus Migrationskontexten

- Wissen um die Medienwelten von Jugendlichen aus Migrationskontexten (nicht nur die deutschsprachige und globale Medienlandschaft, auch andere kulturelle Kontexte und Genres (z. B. Hindi-Filme, Telenovelas, Mangas, Arabesk-Musik etc.)

- Interkulturelle Kompetenzen

- Kompetenzen im Umgang mit «originellem» Verhalten und Verhaltensauffälligkeiten

- Selbstreflexivität: Wie wird die Rolle des Forschers / des Medienpädagogen von den verschiedenen beteiligten Akteuren wahrgenommen (Mitglieder des Medienclubs, Eltern, Lehrpersonal an der Schule)? ${ }^{4}$

- Organisationskompetenzen (Veranstaltung von Elternabenden, Präsentationen, Organisation von Ausflügen, Verhandlung mit Institutionen, Umsetzung von Projektideen)

- gute Englischkenntnisse (Kommunikation im Rahmen eines internationalen Kooperationsprojekts, Übersetzung von Intranetbotschaften etc.)

- Netzwerk-Kontakte entwickeln und nutzen: Einbeziehen von Personen mit Kompetenzen, die man selbst nicht hat. Es ist wichtig, sich der eigenen Begrenzungen bewusst zu sein; aber auch zu wissen, wie Kompetenzen von anderen Menschen hinzugezogen werden können

\section{Kompetenzen: Wissenschaftliche Begleitung}

- Antizipation von Verstehensmöglichkeiten im Forschungskontext (Auf welchem Niveau muss ich kommunizieren, damit ich verstanden werde?)

- Wenn möglich Fremdsprachenkenntnisse ${ }^{5}$ Kontakt mit den Eltern, Hilfestellungen beim Sprechen, Analyse von fremdsprachigen Eigenproduktionen, Analyse der fremdsprachigen Intranetkommunikation)

- Einfühlungsvermögen 
- technische Kompetenzen (Auch der Researcher sollte über ein medientechnisches Grundwissen verfügen, um bei Anfragen parallel zum Media-educator beraten zu können; vgl. Holzwarth \& Maurer 2003)

- Selbstreflexivität bezüglich der Forscherrolle (Balance von Nähe und Distanz Reflexion der Selbstbilder und der Fremdbilder bezügliche der eigenen Rolle im Feld) ${ }^{6}$

- Selbstreflexivität bezüglich der eigenen Vorortung (Was bedeutet es, beispielsweise als junger (Alter bzw. Generationenlagerung), weisser (Herkunft), mittelschichtssozialisierter (Milieu) Mann (Geschlecht) zu forschen?)

- Vermittlungs- und Visualisierungskompetenz (z.B. verschiedenen Zielgruppen die Intentionen eines Forschungsprojekts vermitteln)

- medienpädagogische Kompetenzen (In bestimmten Situationen kann es aus praktischen Gründen wichtig sein, die medienpädagogische Begleitung unterstützen zu können) (vgl. Holzwarth \& Maurer 2003)

- hermeneutische Kompetenzen auf verschiedenen Ebenen der Kommunikation (z. B. schwer verständliche Äusserungen im Interview verstehen, soziale Konstellationen, Dynamiken und Verhaltensweisen verstehen)

Für die Zusammenarbeit zwischen MB und WB ist es wichtig, die Statusunterschiede zu reflektieren. In der Regel arbeitet die MB hauptsächlich während der Praxisphase im Projekt, die WB dagegen ist auch davor und danach präsent. Damit sind die beiden Rollen im Projekt - auch was Status und Vergütung angeht - unterschiedlich gewichtet.

Die Zusammenarbeit von Forscher und Medienpädagoge wird im Wesentlichen durch das übergeordnete Verhältnis von Forschung und Medienarbeit strukturiert: Ist die Medienarbeit dem Primat der Forschung untergeordnet (top down), oder folgt die Forschung den Eigendynamiken der Medienpraxis (bottom up)? Aus unserer Sicht sollten je nach Situation die Bedürfnislagen von Forschung, Medienarbeit und Produzenten flexibel ausgehandelt werden.

Für die Verbindung von Forschung und praktischer Medienarbeit lassen sich folgende Erfahrungswerte formulieren:

\section{Verbindung von Themen der Teilnehmenden und Forschungsfragen}

In diesem Kontext ist es wichtig, offen für die Themen und die Bedürfnisse der Teilnehmer zu sein und gleichzeitig die Forschungsfragen nicht aus dem Auge zu verlieren. Als im März 2003 der Irakkrieg ausbrach, fragten wir im Club die Kinder und Jugendlichen nach ihren Meinungen. Sie diskutierten sehr interessiert und wollten mit den Videokameras auf die Strasse hinausgehen, um Menschen zu interviewen. In einem Fall wie diesem ist es wichtig, den Bedürfnissen der Kinder entgegenzukommen, auch wenn eigentlich etwas anderes auf dem Programm gestanden hätte. Offenheit ist ein zentrales Prinzip explorativer eth- 
nographisch orientierter Forschung. Oft können Forschungsfragen auch indirekt thematisiert werden.

Gemeinsame Vorbereitung und Nachbereitung der Treffen (MB und WB) Um eine gelungene Verbindung von Medienarbeit und Forschung zu erzielen, ist es wichtig, die Treffen sorgfältig gemeinsam zu planen und auszuwerten.

\section{Ausreichend Zeit einplanen und Produktionsdruck vermeiden}

Stehen die Medienclubs aufgrund internationaler Absprachen unter Produktionsdruck, kann sich dies leicht auf die Kinder und Jugendlichen auswirken. Um dies zu verhindern, sollte genug Zeit für Medienproduktion und Internetkommunikation eingeplant werden.

Balance zwischen verbindlichen Rahmenbedingungen, die für alle Partnerländer gelten, und Bereichen, die von den lokalen Besonderheiten abhängen

Ein internationales Forschungsprojekt muss beiden Aspekten gerecht werden, den übergreifend verbindlichen Rahmenbedingungen (z. B. aus allen Ländern Vorstellungsvideos, über die sich die Kinder/Jugendlichen kennen lernen können) und den lokalen Besonderheiten. Dieses Spannungsfeld muss immer wieder reflektiert werden.

\section{Balance von Produktorientierung und Prozessorientierung}

In manchen Situationen ist es wichtig, Produktorientierung in den Vordergrund zu stellen, in anderen dagegen muss den Gruppenprozessen Vorrang gegeben werden. Bei Projekten, in denen wie bei CHICAM sehr viel von den Produkten abhängt (Intranetkommunikation, Produkte als Grundlage für Interviews), besteht unter Umständen die Gefahr, Prozesse aus dem Blick zu verlieren.

\section{Balance von unterschiedlichen Forschungsinteressen/-zielen}

Unter Umständen können sich Forschungsziele widersprechen, wie z. B. einerseits Produktionen, die mit wenig Sprache arbeiten und damit interkulturelle Zugänglichkeit bei der Intranetkommunikation wahrscheinlich machen, und andererseits themenbezogene Produktionen, bei denen über Sprache sehr viel Interessantes kommuniziert wird. Balance kann in diesem Kontext heissen, sich nicht einseitig auf das Eine oder das Andere zu beschränken. Auch Medienproduktion und Intranetkommunikation waren zwei zentrale Projektdimensionen, die aufgrund begrenzter zeitlicher Ressourcen ausbalanciert werden mussten.

\section{Balance von Gruppen- und Einzelarbeit}

Bestimmte Produktionen lassen sich sehr gut in der Gruppe realisieren. Manche erfordern sogar die Gruppensituation. Für andere Projekte dagegen eignen sich 
Kleingruppen- oder Einzelarbeit. Für die Forschung ist zu bedenken, dass Einzelmeinungen bei individuellen Produktionen besser zum Ausdruck kommen.

\section{Balance von anspruchsvollen und weniger anspruchsvollen Produktionen}

Um zu interkultureller Kommunikation zu motivieren und um verschiedene Öffentlichkeiten für die Themen von Kindern und Jugendlichen mit Migrationshintergrund zu sensibilisieren, ist es wichtig, Produktionen zu ermöglichen, die als attraktiv wahrgenommen werden und nicht allzu weit von den ästhetischen Standards der Zielgruppen abweichen. Andererseits muss nicht jedes Produkt komplex und aufwändig gemacht sein. Auch Filme, die schnell und mit geringem Aufwand hergestellt werden (z. B. ohne Schnitte), können für die Zuschauer attraktiv sein. Verschiedene Genres oder Media-models unterscheiden sich in Bezug auf die Tolerierbarkeit von Fehlern und geringer Professionalität: Während kleine Fehler (z. B. Hand im Bild, ruckartige Bewegungen) bei Animationsfilmen dem Filmgenuss kaum abträglich sind, werden narrative Inkonsistenzen und filmsprachliche Unerfahrenheit (z. B. unzureichende Auflösung eines Handlungsablaufs) bei kurzen Spielfilmen weniger leicht toleriert. Erfahrungen dieser Art können bei der Balance von anspruchsvollen und weniger anspruchsvollen Produktionen berücksichtigt werden.

\section{Kontextinformationen erheben und gemeinsam rekonstruieren}

Für die Deutung von Eigenproduktionen spielen Kontextinformationen eine zentrale Rolle. Deshalb sollten die Umstände der Produktion so genau wie möglich erhoben werden. An diesem Prozess sollten beide Perspektiven, die der WB und der MB einbezogen werden. Auch medienpädagogische und medienästhetische Inputs sollten gemeinsam reflektiert werden.

\section{Durch subjektadäquate Vermittlung der Intentionen Akzeptanz für die Forschung erlangen}

Für die Präsentation der Projektziele hatten wir mehrere Bilder ausgewählt. Viele Aspekte der Forschung liessen sich leicht visualisieren (z. B. Tower-Bridge als visuelle Veranschaulichung für London, Eine Fotokamera als Zeichen für Fotoarbeit etc.). Besonders schwierig war es, den Aspekt Forschung zu visualisieren. Wir wählten ein Buch und eine Brille als Symbol. Es war uns klar, dass die Verdeutlichung der Forschungsdimension ein Prozess sein würde und dass wir Forschung an verschiedenen Punkten immer wieder auf unterschiedliche Arten erklären würden. Gute Erfahrungen machten wir mit der Strategie, Eltern und Kindern bei Familienbesuchen die Buchpublikation eines früheren Projektes (VideoCulture) mitzubringen (Niesyto 2003). So konnten wir das Buch mit seinen Texten und Bildern präsentieren und erklären, dass CHICAM ein ähnliches Projekt ist, über das ein Buch geschrieben werden wird, in dem auch Bilder ent- 


\begin{tabular}{|c|c|}
\hline Medienpädagogische Aktivitäten & Forschungsbereiche \\
\hline $\begin{array}{l}\text { Medienkollagen mit Fotos und Ausschnitten von Zeit- } \\
\text { schriften (Jugendzeitschriften und andere Magazine) }\end{array}$ & $\begin{array}{l}\text { - Medienerfahrungen und -präferenzen } \\
\text { - Selbstdarstellungswünsche }\end{array}$ \\
\hline Einwegkamerabilder zum Thema Tagesablauf ${ }^{7}$ & $\begin{array}{l}\text { - Informationen über wichtige Bezugspersonen, } \\
\text { - Aktivitäten und Orte. } \\
\text { Die Bilder können als Grundlage für Interviews } \\
\text { dienen («photo-elicitation») }\end{array}$ \\
\hline Einwegkamerabilder zum Thema Freundschaft & $\begin{array}{l}\text { - Informationen über Freundschaft (Alter, Ge- } \\
\text { schlecht, kulturelle Hintergründe) }\end{array}$ \\
\hline Einwegkamerabilder zum Thema Familie & - Informationen über familiäre Kontexte \\
\hline $\begin{array}{l}\text { Die Kinder/Jugendlichen bitten, Fotografien aus ih- } \\
\text { ren Herkunftsländern mitzubringen und sie zu einem } \\
\text { kurzen Clip mit Musik bzw. Kommentar zu arrangieren } \\
\text { (〈picture show })^{8}\end{array}$ & $\begin{array}{l}\text { - Informationen über Erfahrungen der Kinder/Ju- } \\
\text { gendlichen im Herkunftsland und über subjektive } \\
\text { Erinnerungskultur bzw. «memory management` }\end{array}$ \\
\hline $\begin{array}{l}\text { Die Kinder/Jugendlichen bitten Objekte aus ihren } \\
\text { Herkunftsländern mitzubringen und sie zu einem kur- } \\
\text { zen Clip mit Musik bzw. Kommentar zu arrangieren }{ }^{10}\end{array}$ & - siehe oben \\
\hline $\begin{array}{l}\text { Foto-Essay über Dinge, die die Kinder/Jugendlichen } \\
\text { mögen bzw. nicht mögen }\end{array}$ & $\begin{array}{l}\text { - allgemeine Orientierung, kulturelles Kapital, Ge- } \\
\text { schmack }\end{array}$ \\
\hline
\end{tabular}

Tabelle 1: Beispiele für medienpädagogische Aktivitäten und Forschung

halten sind. Insbesondere am Beispiel der Praxisdimension konnten wir Eltern und Kindern zeigen, dass es uns darum geht, anderen Menschen Anregungen zu geben, damit ähnliche Medienprojekte auch in Zukunft stattfinden werden. «lch schreibe ein Buch über junge Menschen, die nicht in Deutschland geboren sind» erschien als Erklärung in vielen Kontexten (z. B. auch in deutschsprachigen nicht-akademischen Milieus) angebracht.

- Differenzierung zwischen Forschung, die im Rahmen der Medienarbeit erfolgen soll und Forschung, die unabhängig von Medienarbeit (bei Extratreffen ausserhalb der Medienclubs) erfolgen kann.

Manche Daten liessen sich nur im Clubkontext erheben, für andere dagegen war es sinnvoll, Extratreffen zu vereinbaren. Hätte beispielsweise die gesamte Intranetkommunikation im Rahmen der eigentlichen Clubtreffen stattgefunden, wäre ein ernsthaftes Zeitproblem entstanden. Aktivitäten ausserhalb der Medienarbeit wie z. B. Ausflüge, Kinobesuche oder Picknicks sind wichtige informelle Datenerhebungssituationen. 
Die folgende Übersicht zeigt Projektbeispiele für die Verknüpfung von praktischer Medienarbeit im Clubkontext und Forschung:

Neben dem Clubkontext und den Extratreffen waren auch «in-between-settings wichtig:

«In between settings - that is, opportunities for observation that arise in the spaces between structured activities or institutional contexts - are, in the partners' experience, particularly fruitful when working with groups of this kind...» (de Block et al. 2004, p. 3).

Im Rahmen der Forschung ergaben sich diverse informelle Erhebungssituationen, wie z. B. im Schulhof, in den Pausen zwischen zwei Unterrichtsstunden, vor und nach den CHICAM-Clubtreffen, bei gemeinsamen Ausflügen im Schulkontext und im CHICAM-Kontext sowie auf dem Weg zu Drehorten. Manchen Kindern/Jugendlichen kamen solche informellen Erhebungssituationen mehr entgegen als die offizielleren Settings. Eine Teilnehmerin beispielsweise äusserte sich offen in diesen Situationen, schien aber weniger Lust auf Interviewsituationen mit offiziellem Charakter und laufendem Mikrophon zu haben. Gespräche mit den Eltern hatten durchweg informellen Charakter - laufende Mikrophone wären aus unserer Sicht erst bei einer stärker entwickelten Forschungsbeziehung sinnvoll gewesen.

\section{Resümee}

Das Arbeiten mit visuellen Medien wie im Projekt CHICAM birgt in verschiedenerlei Hinsicht Chancen für die Forschung und für die Praxis: Über Fotos, die Subjekte selbst anfertigen, können Forschende wichtige Hinweise auf bedeutsame Aspekte der Lebenswelt erhalten (heuristische Funktion). In gewisser Weise ermöglichen visuelle Methoden privilegierte subjektnahe Zugänge zu lebensweltlichen Aspekten. Da visuelle und audiovisuelle Medien eine wichtige Rolle im Leben von Kindern und Jugendlichen spielen, bedeutet die Anwendung visueller Forschungsansätze auch eine hohe Subjektadäquatheit.

Selbstproduzierte Fotos erleichtern Kommunikation bei Interviews («photo-elicitation〉). Dies ist vor allem dann wichtig, wenn - wie bei CHICAM - sich die Subjekte nicht in ihrer Muttersprache äussern können oder sonstige sprachliche Einschränkungen vorliegen. Oft lassen sich schwierige und problematische Aspekte mit visuellen Methoden eher oder leichter thematisieren. Kinder und Jugendliche können im Rahmen von Medien-Eigenproduktionen bis zu einem gewissen Grad selbst entscheiden, was sie aus ihrer Lebenswelt zeigen möchten («participatory research»).

Die Verbindung von Medienarbeit und Praxisforschung trägt dazu bei, dass der Forschungsprozess durch einen Charakter des gegenseitigen Gebens und Nehmens geprägt wird. Die am Projekt beteiligten Kinder geben Einblicke in ihre 
Lebenswelt. Die beteiligten Erwachsenen geben Aufmerksamkeit, vermitteln Medienkompetenz und - sofern gewisse didaktische Prinzipien berücksichtigt werden - ermöglichen Spass und neue (Lern-) Erfahrungen. Im Umgang mit Medien können die Beteiligten Selbstwirksamkeit erfahren und ihr Selbstwertgefühl entwickeln (z. B. Menschen auf der Strasse ansprechen, mit dem Medium Video neue Räume erkunden). Durch Medienarbeit kann Kontakt zwischen Migranten und Nicht-Migranten entstehen beziehungsweise hergestellt werden (z. B. Präsentation von Medienprodukten). Selbstausdruck mit audiovisuellen Medien kann ausserdem sprachliche Defizite kompensieren; dabei verbindet Medienarbeit Medienkompetenz und Sprachkompetenz.

Forschungsdesigns, die die Erhebung umfangreichen Kontextmaterials ermöglichen, bereichern die sozialwissenschaftliche Interpretation visueller Daten, indem bestimmte Lesarten durch weiteres Material gestützt werden können. Damit ermöglichen Kontextinformationen die Entwicklung von Lesarten und Deutungsperspektiven, die dem Forscher ansonsten verschlossen bleiben würden.

\section{Literatur}

Baacke, Dieter / Sander, Uwe / Vollbrecht, Ralf (Hg.): Lebenswelten sind Medienwelten. Opladen: Leske und Budrich, 1990.

Buckingham, David / Grahame, Jenny / Sefton-Green, Julian: Making media. Practical producing in media education. London: The English and Media Center, 1995.

de Block, Liesbeth / Buckingham, David / Holzwarth, Peter / Niesyto, Horst: Visions Across Cultures: Migrant Children Using Audio-Visual Images to Communicate. Children in Communication about Migration (CHICAM). Deliverables 14 and 15. August 2004 (http://www.chicam.net/reports/ download/visions_across_cultures.pdf> (20.9.2006)

Dubs, Rolf: Konstruktivismus; einige Überlegungen aus der Sicht der Unterrichtsgestaltung. In: Zeitschrift für Pädagogik 41(1995), S. 889-903.

Friedrich, Helmut / Eigler, Gunter / Mandl, Heinz / Schnotz, Wolfgang / Schott, Franz / Seel, Norbert M. (Hg.): Multimediale Lernumgebungen in der Betrieblichen Weiterbildung. Gestaltung, Lernstrategien und Qualitätssicherung. Neuwied: Luchterhand, 1997.

Holzwarth, Peter; Maurer, Björn: Aesthetic Creativity, Reflexivity and the Play with Meaning: a VideoCulture Case Study. In: Kirkwood, Adrian (Hg.): Journal of Educational Media. Vol. 26, No. 3, 2001, p. 185-202.

Holzwarth, Peter; Maurer, Björn: CHICAM (CHILDREN IN COMMUNICATION ABOUT MIGRATION): An international research project exploring the possibilities of intercultural communication through children's media productions. In: Kiegelmann, Mechthild; Gürtler, Leo (Eds.): Research Questions and Matching Methods of Analysis. Qualitative Psychology Nexus: Vol. 3. Tübingen: Ingeborg Huber, 2003, p. 125-139. 
Marotzki, Winfried; Niesyto, Horst (Hg.): Bildinterpretation und Bildverstehen. Methodische Ansätze aus sozialwissenschaftlicher, kunst- und medienpädagogischer Perspektive. Wiesbaden: VS Verlag für Sozialwissenschaften, 2006.

Maurer, Björn: Medienarbeit mit Kindern aus Migrationskontexten. Grundlagen und Praxisbausteine. München: kopaed, 2004.

Niesyto, Horst: (Hg.): VideoCulture. Video und interkulturelle Kommunikation. München: kopaed, 2003.

Niesyto, Horst: Eigenproduktionen mit Medien als Gegenstand der Kindheits- und Jugendforschung. Einleitende Bemerkungen zu den methodologischen Herausforderungen einer Forschungsperspektive. In: Niesyto, Horst (Hg.): Selbstausdruck mit Medien. Eigenproduktionen mit Medien als Gegenstand der Kindheits- und Jugendforschung. München: kopaed, 2001c, S. 7-14.

Niesyto, Horst: Jugendforschung mit Video. Formen, Projekte und Perspektiven eines Forschungsansatzes. In: Niesyto, Horst (Hg.): Selbstausdruck mit Medien. Eigenproduktionen mit Medien als Gegenstand der Kindheits- und Jugendforschung. München: kopaed, 2001a, S. 89-102.

Niesyto, Horst: VideoCulture - Gegenstand, Methoden, Ergebnisse. In: Niesyto, Horst (Hg.): Selbstausdruck mit Medien. Eigenproduktionen mit Medien als Gegenstand der Kindheits- und Jugendforschung. München: kopaed, 2001b, S. 157-172.

Schell, Fred: Aktive Medienarbeit mit Jugendlichen. Theorie und Praxis. 3. Aufl. München: kopaed, 1999.

Wicke, Rainer-Ernst: Grenzüberschreitungen. Der Einsatz von Musik, Fotos und Kunstbildern im Deutsch-als-Fremdsprachunterricht. In: Schule und Fortbildung. München: iudicium, 2000.

\section{Fussnoten}

1 Mehr Informationen (Projektergebnisse, Hinweise für die Praxis und policy-recommendations) siehe unter: 〈http://www.chicam.org sowie http:// ph-ludwigsburg.de/2228.html>. Das Projekt CHICAM wurde von Prof. Dr. David Buckingham und Dr. Liesbeth de Block (University of London, Institute of Education) koordiniert. In Deutschland lag die Projektleitung bei Prof. Dr. Horst Niesyto (PH Ludwigsburg, Abteilung Medienpädagogik); der lokale CHICAM-Club wurde von Dipl.-Päd. Peter Holzwarth (wissenschaftlicher Mitarbeiter) und von Dipl.-Päd. Björn Maurer (Medienpädagoge) begleitet. Die Finanzierung von CHICAM erfolgte durch das 5. Rahmenforschungsprogramm der EU.

2 In Griechenland fand der CHICAM-Club im Kontext einer Flüchtlingsorganisation statt.

3 In der CHICAM Pilotphase machten wir die Erfahrung, dass die Kinder das (gestalterische) Interesse verlieren, wenn bspw. Aufnahmen, die sie gemacht haben, aus Zeitgründen anschliessend nicht mehr angeschaut werden können.

4 Supervision im Kontext von qualitativer Forschung kann sinnvoll sein, um soziale Interaktionen im Forschungsprozess systematischer reflektieren 
zu können und um allgemein die Qualität der Forschungsarbeit zu erhöhen. Wir konnten während unserer Praxisphase sehr gute Erfahrungen mit Forschungssupervision machen. Besonders hilfreich war, dass unsere Supervisorin bereits mit einer ähnlichen Zielgruppe (Schülerinnen und Schüler aus Migrationskontexten) zusammengearbeitet hatte. Dadurch ergab sich ein vertieftes Verständnis bestimmter Konstellationen.

5 Fremdsprachenkenntnisse können in verschiedenen Forschungskontexten von Bedeutung sein. Sie erleichtern die Kommunikation mit den Eltern, unter Umständen können in Interviews Missverständnisse geklärt werden und Videoproduktionen und Intranet-Kommunikation können leichter verstanden werden. Fremdsprachen ermöglichen auch Zugänge zum jeweiligen Herkunftsland. Unter Umständen können mit dem Beherrschen von wenigen wichtigen Worten einer Fremdsprache (Formen der Begrüssung, des Dankes und des Bittens) auch Respekt, Interesse und Anerkennung ausgedrückt werden. Es sollte auch bedacht werden, dass für Erwachsene häufig auch Jugendsprachen Fremdsprachen darstellen.

6 In diesem Kontext steht auch die Reflexion möglicher Übertragungsprozesse (z. B. aufgrund der Unbekanntheit der Forscherrolle wird die bekanntere Rolle des Lehrers aktiviert).

7 Vgl. Videobeispiel: 〈http://www.chicam.org/videos/media/germany/ slideshow.html> [Zugriffsdatum: 8.6.2007]

8 Vgl. Videobeispiel: 〈http://www.chicam.org/videos/media/netherlands/ sharmakes_family.html> [Zugriffsdatum: 8.6.2007]

9 Der Begriff «memory management» stammt aus dem Computerbereich. In unserem Kontext soll er - analog zum musikwissenschaftlichen Konzept «mood management» - den Prozess des aktiven Gestaltens von persönlicher Erinnerung beschreiben. Dieses aktive Moment des Erinnerns drückte auch der Fotograf Duane Michals in seinem Zitat «Past is what you choose to remember.» aus (Staatliche Akademie für Bildende Künste Stuttgart, April 1996).

${ }^{10} \mathrm{Vgl}$. Videobeispiel: 〈http://www.chicam.org/videos/media/italy/oggetto_del_mio.html> [Zugriffsdatum: 8.6.2007] 